\title{
INTEGRATED SIMULATION MODEL FOR COMPOSITION AND PROPERTIES OF GASES IN HYDROGEN FUELED ENGINE
}

\author{
Mohammed Kamil ${ }^{1,2}$, M.M. Rahman ${ }^{1,3^{*}}$ and Rosli A. Bakar ${ }^{1,3}$ \\ ${ }^{1}$ Faculty of Mechanical Engineering, Universiti Malaysia Pahang \\ 26600 Pekan, Pahang, Malaysia \\ *E-mail: mustafizur@ump.edu.my \\ Phone: +6094246239; Fax: +6094246222; \\ ${ }^{2}$ Faculty of Engineering, University of Tikrit, Tikrit, Iraq \\ ${ }^{3}$ Automotive Engineering Centre, Universiti Malaysia Pahang \\ 26600 Pekan, Pahang, Malaysia.
}

\begin{abstract}
The composition and thermodynamic properties of combustion products are essential for evaluating the performance of internal combustion engines. This paper presents a simulation model named 'HydEnPro', to predict the composition and thermodynamic properties of the multi-component gases in hydrogen fueled engines. HydEnPro was developed as a set of codes for predicting the chemical equilibrium composition with two databases (NASA and Chemkin) that contain the thermodynamic information about the molecules. The developed codes accessed the thermodynamic databases and equilibrium codes predicted the properties of the multi-component gases. The predictions of HydEnPro were validated against the well-recognized Chemkin tables, the standard literature and other general simulation models. It is shown that HydEnPro translation for the databases and its interfaces are correctly implemented. In addition, the accuracy of HydEnPro was emphasized. Hence, HydEnPro can be used in a predictive manner.
\end{abstract}

Keywords: Hydrogen fuel; hydrogen engine; chemical equilibrium; hydrogen combustion products.

\section{INTRODUCTION}

Non-OPEC oil-producing countries are already approaching their peak production, leaving most of the remaining reserves in the politically unstable Middle East (Campbell, 2000; Pfeiffer, 2006), and the new energy regime has the potential to remake civilization along radical new lines. Hydrogen is the most basic and ubiquitous element in the universe. It never runs out and produces no harmful $\mathrm{CO}_{2}$ emissions when burned; the only by-products are heat and pure water. A new economy powered by hydrogen will fundamentally change the nature of our market, political, and social institutions, just as coal and steam power did at the beginning of the industrial age (Verhelst \& Wallner, 2009; Sopena et al., 2010; Kelly et al., 2010; Lee et al., 2010; Hamada, Rahman, and Aziz, 2012a,b). It is well recognized by governments, academic institutions, associations, and industry that our window of opportunity for a managed transition to alternative energy resources has been shortened. Hence, there is huge momentum in hydrogen fuel research (Rahman et al., 2009a,b, 2010, 2011a,b, 2013; Hamada et al., 2012, 2013; Kamil et al., 2012). Hydrogen fuel utilization in internal combustion engines (ICE) has attracted a considerable portion of this research. The 
majority of the research in hydrogen fueled engines $\left(\mathrm{H}_{2} \mathrm{ICE}\right)$ has been in experimental studies (Kamil et al., 2011; Rahman et al., 2013; Hamada et al., 2012, 2013). However, simulation models for ICEs play a vital role in allowing the automotive engineer to evaluate complex operation strategies that cannot be analyzed directly with other means. Besides, the simulation models allow the engineer to analyze many alternative strategies quickly and avoid the risk, expense and disruption associated with extensive laboratory experimentation.

To develop an integrated model for the engine, it is essential to specify the composition and properties of the multi-component gas mixture at every stage of the simulation. Hence, a gas mixture model is a crucial part that must be incorporated within the main model. Several equilibrium codes for specifying the species of equilibrium combustion products and calculating their thermodynamic properties have been developed and used among researchers in the combustion community, and some are available for open use. The most well-known codes are Villars-Cruise-Smith (VCS), an algorithm developed and modified by Villars (1959), Cruise (1964), and Smith and Missen (1968); STANJAN, a code developed by Olikara and Borman (1975); and the NASA-Lewis code developed by Gordon and McBride (1976). Even though the VCS code was developed for analyzing military gun propellants, it can be applied to a wide variety of problems. This code is dimensioned to include as many as 700 species in a calculation. Furthermore, different models for the gaseous mixture can be used in the code. The gaseous products can be assumed as a mixture of perfect gases, an ideal mixture of imperfect gases or a non-ideal mixture of imperfect gases, which is the most general mode1 for a gas mixture. However, ICE application does not require such computationally intensive and time-consuming code. The NASA-Lewis code is also an extensive code; it is capable of solving equilibrium problems for several phases and for over 100 species. This code is also more comprehensive and too computationally intensive. It is anticipated that it would be more complex to learn and incorporate. On the other hand, the STANJAN program is faster than the NASA-Lewis code because it was written specifically for engine combustion with a reduced set of species, and is shorter and easier to understand. STANJAN code was written in FORTRAN at Stanford University. It is distributed freely for research purposes as a compiled code. But the compiled version requires a user interface, which cannot be called a subroutine by another program. There is no model easily available written in MATLAB which simulates the equilibrium composition and properties for ICE, in particular for $\mathrm{H}_{2} \mathrm{ICE}$ applications. Therefore, a gas mixture composition and property model for $\mathrm{H}_{2} \mathrm{ICE}$ applications is developed. A simulation program model, named HydEnPro is developed in MATLAB as an implementation to the developed model. HydEnPro calculates the chemical equilibrium and thermodynamic properties of reactants and products of a combustion reaction between hydrogen fuel and air.

\section{THERMODYNAMIC PROPERTIES}

For $\mathrm{H}_{2} \mathrm{ICE}$, as well as other fueled engines, the gas properties of interest are: specific heats, $C_{p}$ and $C_{v}$; specific heat ratio, $\gamma$, gas constant, $R$; molecular weight $M$; specific enthalpy, $h$; specific entropy, $s$; specific volume, $V_{s p c}$ and specific internal energy, $u$. In the context of the current simulation, these properties are modeled on both mass and mole bases. This multifarious representation of properties gives extra flexibility when dealing with the different databases. Some databases report their data on a mass basis, others on a molar basis. In addition, the properties can be calculated in the different 
parts of the engine (intake and exhaust manifolds, cylinders, etc.), as well as during the individual processes (intake, expansion, compression and exhaust) at any instant (in crank angle domain or time domain). The bridgehead was identifying the nature of the mixture in the different processes, since the composition of the mixture changes during the engine operating cycle. Table 1 summarizes the considered mixture constituents for $\mathrm{H}_{2}$ ICE. A light was sought from the analysis presented by Heywood (1988) for conventional fuels. Thermodynamic properties for the combustion reactants and products are available as tabulated data. JANAF Thermochemical Tables are the main source of these data (NBS, 1971). However, for computer codes, it is awkward to deal with tabular data. Gordon and McBride (1976) fitted the tabulated JANAF data to polynomials by minimizing the least squares error, and calculated the properties as functions of temperature. These polynomials were known as NASA Polynomials. Similar procedures were followed by Kee, Ripley, and Miller (1991) to produce Chemkin Polynomials. Therefore, four sets of coefficients are considered here for each constituent. Those sets are given over two adjacent temperature intervals: 300 to $1000 \mathrm{~K}$ and 1000 to $5000 \mathrm{~K}$; and for either the NASA or the Chemkin Polynomials.

Table 1. Gas constituents in $\mathrm{H}_{2} \mathrm{ICE}$.

\begin{tabular}{ll}
\hline Process & Constituents \\
\hline Intake & $\begin{array}{l}\text { Hydrogen, air, } \\
\text { residual gas (within the cylinder) }\end{array}$ \\
Compression & Hydrogen, air, residual gas \\
Expansion & $\mathrm{H}_{2} \mathrm{O}, \mathrm{N}_{2}, \mathrm{O}_{2}, \mathrm{H}_{2}, \mathrm{H}, \mathrm{O}, \mathrm{OH}$ and NO. \\
Exhaust & $\mathrm{H}_{2} \mathrm{O}, \mathrm{N}_{2}$ and either: $\mathrm{O}_{2}$ when $\phi<1$ or $\mathrm{H}_{2}$ when $\phi>1$ \\
\hline
\end{tabular}

\section{VALIDATION OF HydEnPro MODEL}

The most important validation of HydEnPro is compared with the well-recognized Chemkin Tables (Kee et al., 1991). The results of HydEnPro are evaluated with respect to how well it can reproduce figures that appear in the standard literature. Some validations are made with the available models, such as chemical equilibrium in perfect gases code (Gaseq, 2011) and the adiabatic flame temperature program (AFTP, 2012). Figure 1 shows $C_{p}$ of six individual species $\left(\mathrm{H}_{2} \mathrm{O}, \mathrm{N}_{2}, \mathrm{O}_{2}, \mathrm{H}_{2}, \mathrm{OH}\right.$, and $\left.\mathrm{NO}\right)$ produced from high temperature combustion reaction in the temperature interval of 1000-3000 K. The results of HydEnPro (solid lines) are drawn together with Chemkin data (Kee et al., 1991) (symbols). The differences are minor and the agreement is perfect. Furthermore, Turns (2006) reported that the specific heat of the $\mathrm{H}$-atom is constant $(=20.786$ $\mathrm{kJ} / \mathrm{kmole} \mathrm{K}$ ) in the temperature range of $200-5000 \mathrm{~K}$, which agrees with the current results. This adds additional confirmation for the validity of HydEnPro. Figure 2 presents a comparison of the enthalpy results obtained by HydEnPro and by Chemkin data for a high temperature combustion reaction. It shows perfect agreement with Chemkin data for all the considered species. Chemkin data tables (Kee et al., 1991) give enthalpy values as a difference between the required values and enthalpy of formation at $298 \mathrm{~K}$. Therefore, the corresponding enthalpy of formation at 298 was added to the given value.

Figure 3 illustrates the entropy values of the individual species produced from high temperature combustion. The results calculated by HydEnPro are displayed together with the values from Chemkin data. The variations within the temperature 
interval of $1000-3000 \mathrm{~K}$ were also demonstrated. The deviation is negligible and HydEnPro shows its validity. Figure 4 evaluates the validity of HydEnPro for low temperature mixtures. This figure collects the results of enthalpy from HydEnPro and Chemkin data together at a temperature range of 300-1000 K. The results for $\mathrm{H}_{2} \mathrm{O}$ are presented due to its different values. It is concluded that the HydEnPro results at a low temperature range agree perfectly with the well-reviewed literature. Specific heat, enthalpy and entropy are selected for validation purposes, since their rule is central in all chains of the modeled thermodynamic relationships. Any one of the considered properties is related to them in some way. This can be clarified further by reviewing the relationships developed in the previous section.

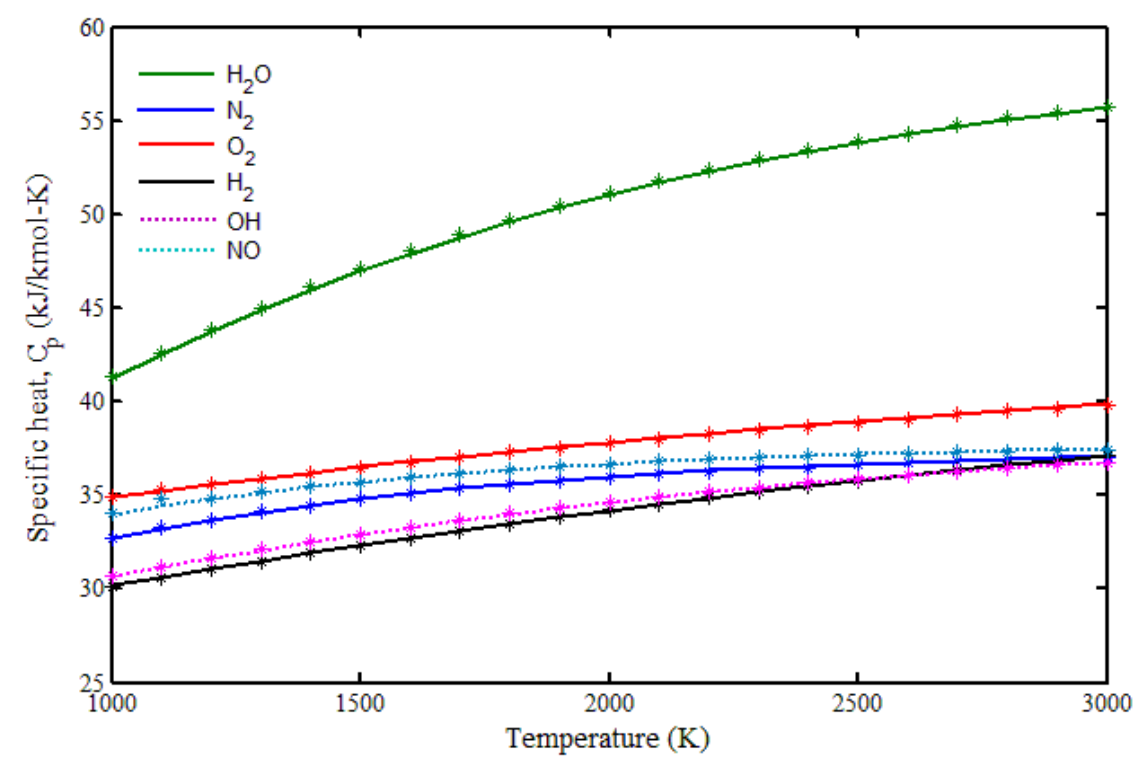

Figure 1. Specific heat $\left(C_{p}\right)$ for the combustion products. Symbols denote Chemkin data (Kee et al., 1991). Values of $\mathrm{H}$ are constant $(=20.785 \mathrm{~kJ} / \mathrm{kmol} \mathrm{K}$ from HydEnPro; and $=20.786 \mathrm{~kJ} / \mathrm{kmol} \mathrm{K}$ in Chemkin). Values of $\mathrm{O}$ are almost constant $(=20.859 \pm 0.084$

$\mathrm{kJ} / \mathrm{kmol} \mathrm{K}$ from HydEnPro; and $=20.859 \pm 0.084 \mathrm{~kJ} / \mathrm{kmol} \mathrm{K}$ in Chemkin).

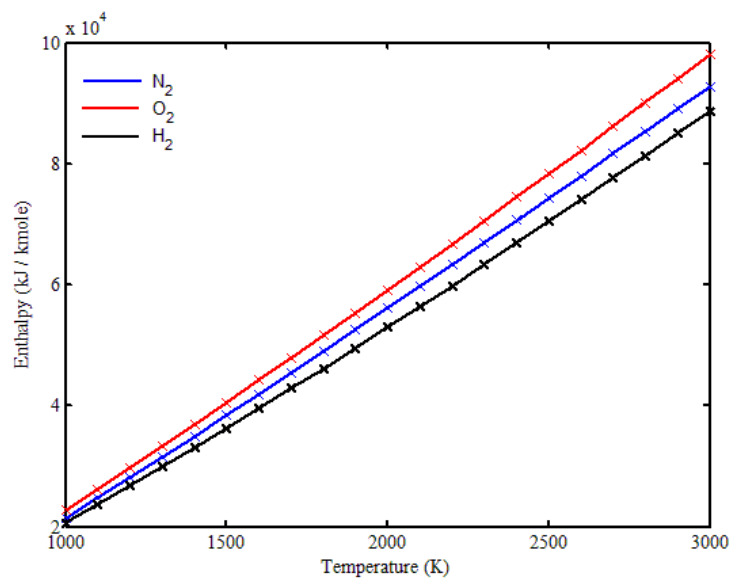

(a)

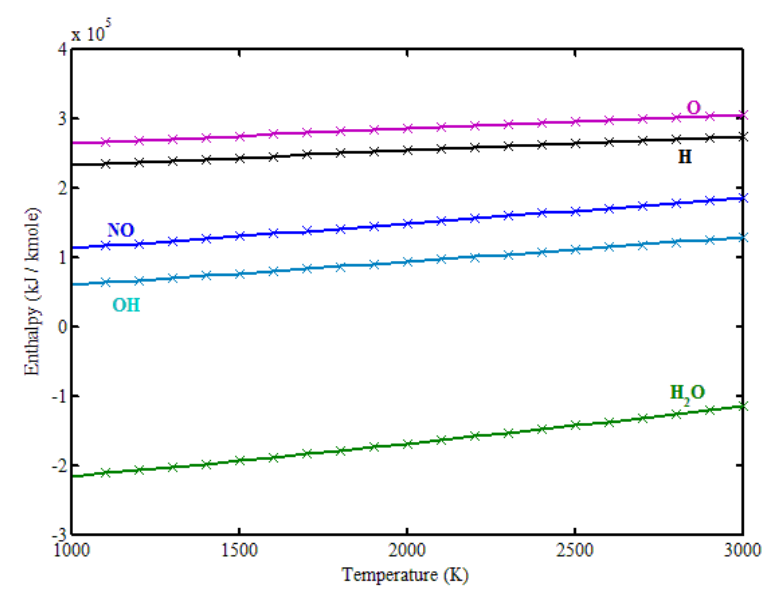

(b)

Figure 2. Variation of species enthalpy. Symbols denote Chemkin data. 


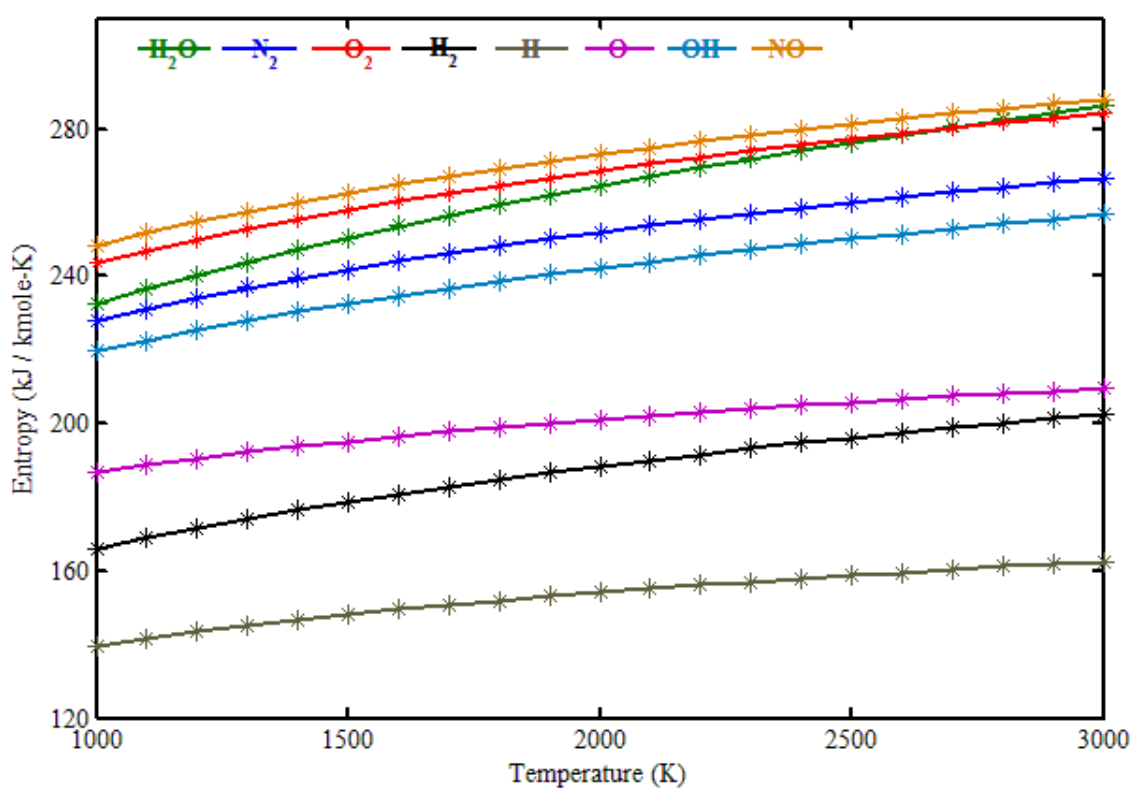

Figure 3. Variation of entropy for the combustion products.

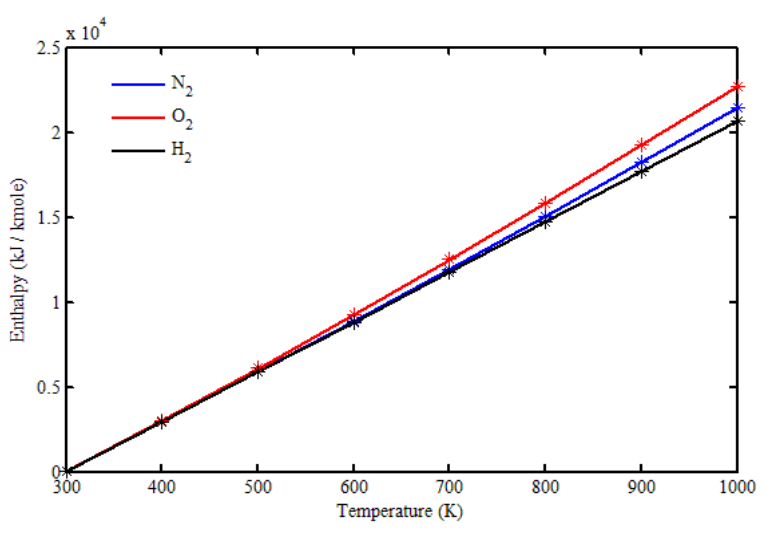

(a)

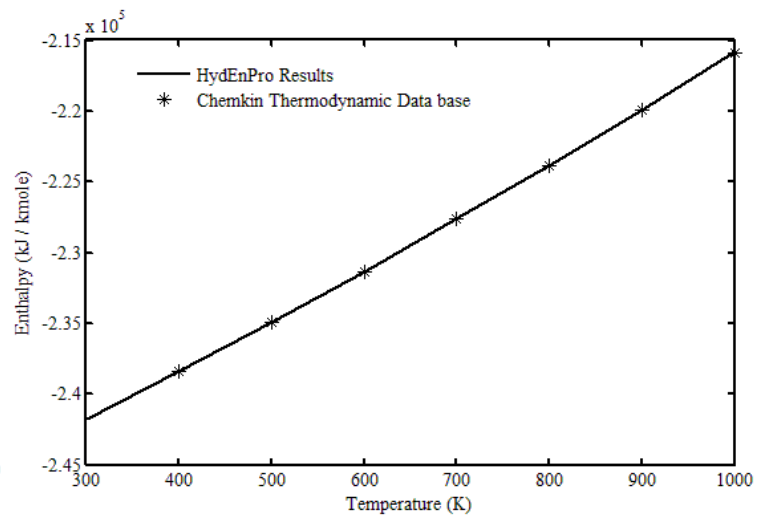

(b)

Figure 4. Variation of enthalpy for low temperature combustion products. Symbols denote Chemkin data (Kee et al., 1991).

Adiabatic flame temperature estimation was validated with results produced by the codes: Gaseq (2011) and AFTP (2012). Figure 5 depicts the variation of $T_{a d}$ with $\phi$ for three different mixture temperatures: 500, 700 and $900 \mathrm{~K}$. The results of HydEnPro, Gaseq and AFTP are plotted together. Some points can be concluded readily; there is apparent coincidence among the three curves in the lean zone $(\phi<1)$ until the stoichiometric condition $(\phi=1)$. Besides, the results of HydEnPro and Gaseq seem to be more nearly identical compared with the AFTP results. The third point is that more correspondence between the three curves is seen as the initial temperature increases. This difference occurs because AFTP considers 30 constituents, while HydEnPro considers only eight. The calculations of Gaseq were limited to the same eight species, but this cannot be done with AFTP. As illustrated, the species considered by HydEnPro are the most common for hydrogen fuel burning in internal combustion engine 
applications. Fortunately, the deviation occurs in the rich region $(\phi \geq 1.2)$, where it is not possible to get stable operation for a hydrogen engine, because of the hydrogen combustion anomalies (knocking, pre-ignition and backfire). The authors could not find any experimental work in the literature which considers this region because pre-ignition, backfire and knocking problems become more serious in the vicinity of stoichiometric conditions.

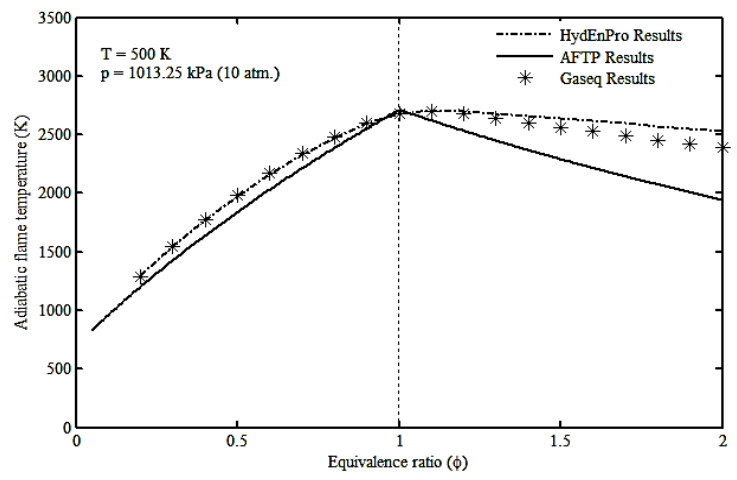

(a)

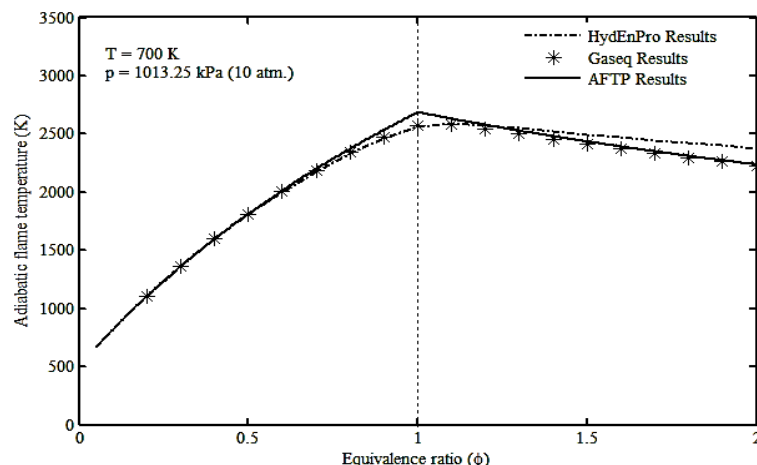

(b)

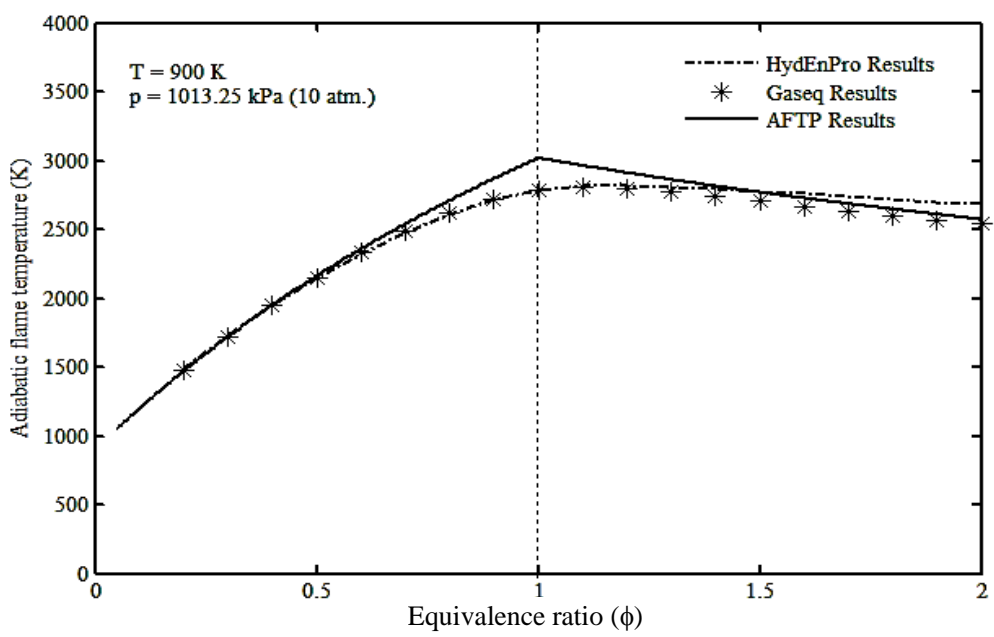

(c)

Figure 5. Adiabatic flame temperature as a function of fuel/air equivalence ratio.

\section{RESULTS AND DISCUSSION}

\section{Assessment of HydEnPro Multi-choices}

Figure 6 shows the comparison between the composition results calculated by using the two considered data bases: NASA and Chemkin. This figure indicates that the difference between them is insignificant. Therefore, the equilibrium compositions, and consequently the properties, calculated by the two different databases will be practically the same. Figure 7 presents the difference between the results of the two equilibruim codes: eqp_9 and eqp_4. The performance of the two codes from the accuracy point of veiw is the same because their results are exactly the same and the differences are negligible. However, the time consumed by eqp_9 and eqp_4 for doing the same 
calculations is different, as shown in Figure 8. The central processing unit time for calculations at different equivalence ratios is given there. In the majority of the cases, the time consumed by eqp_4 is less than or equal to that of eqp_9. In fact, for applications like the control application, this time is something that must not belittled.

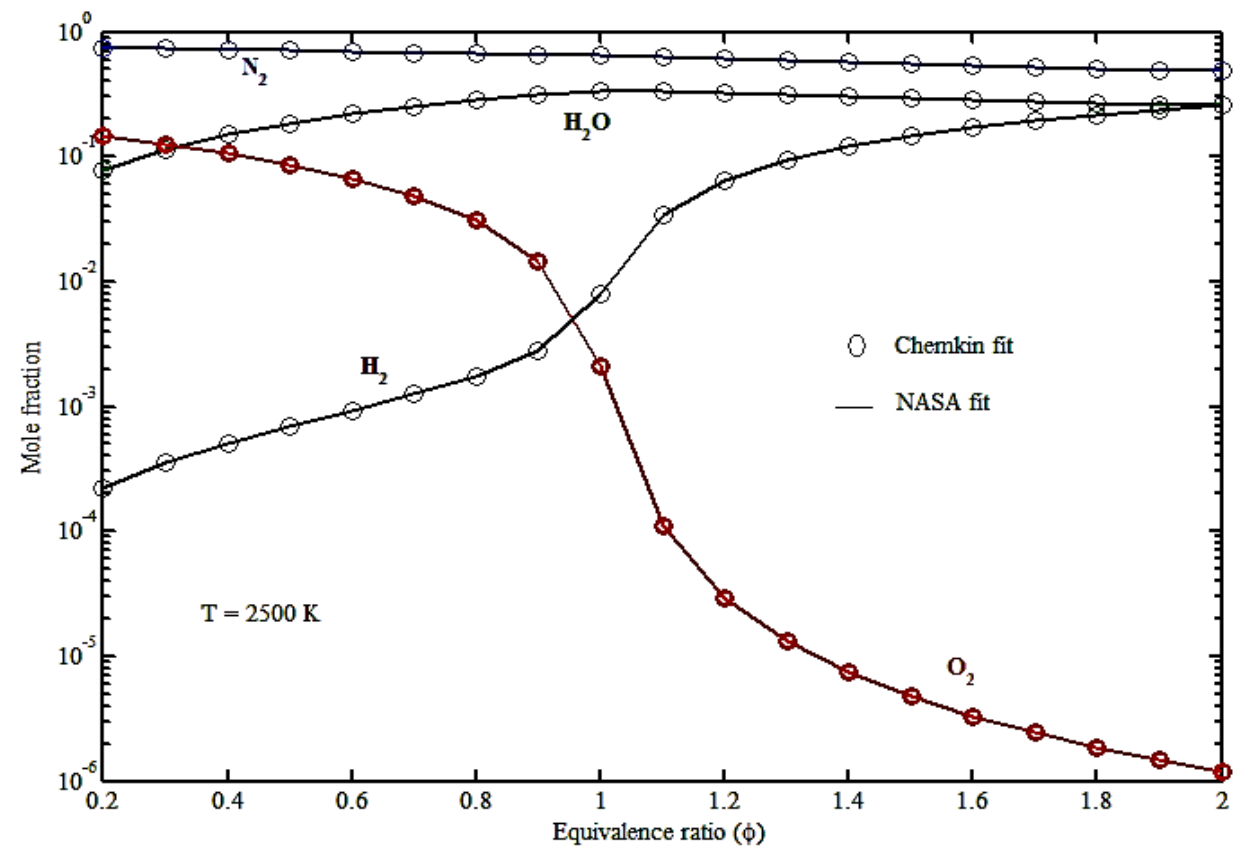

(a)

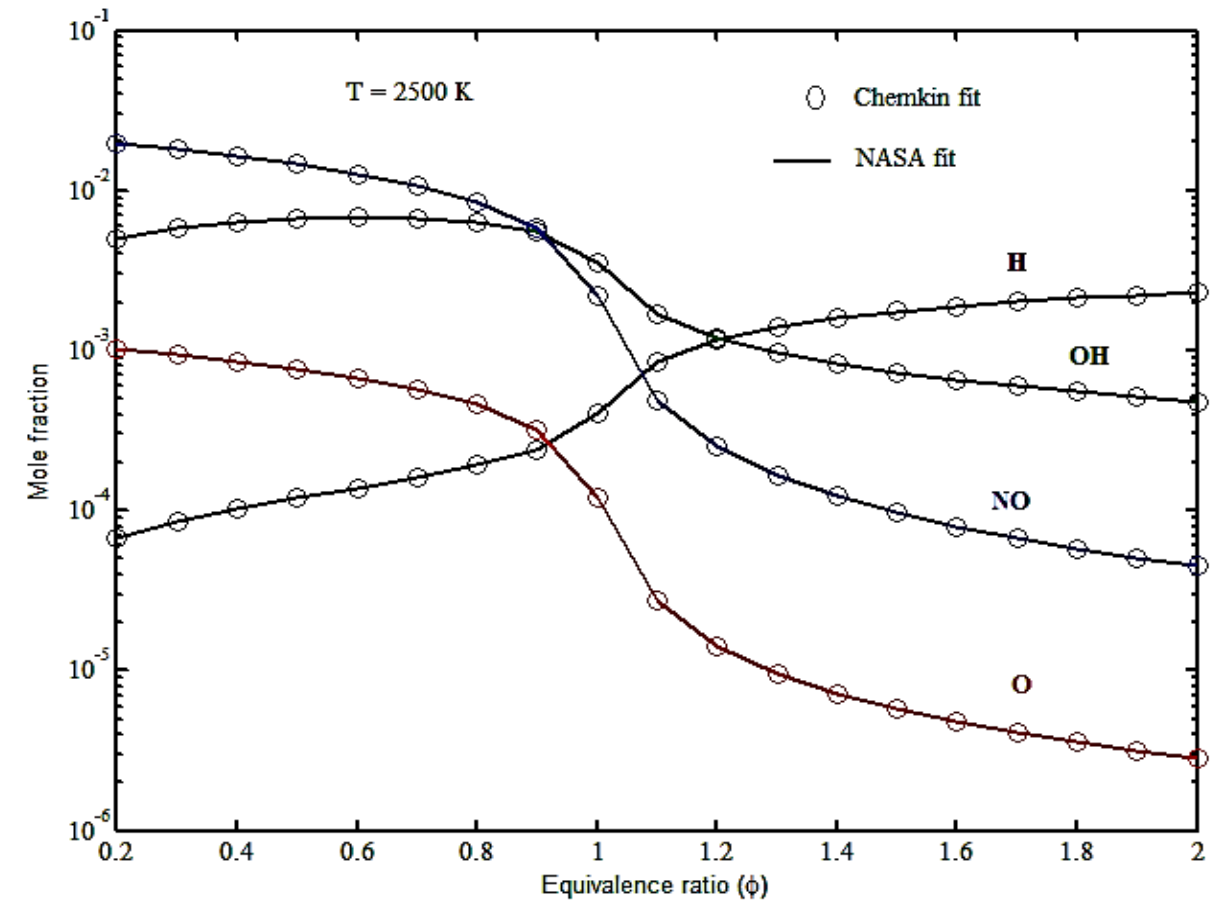

(b)

Figure 6. Comparison between NASA fit and Chemkin fit: mole fraction as a function of $\phi$ at $2500 \mathrm{~K}$. 


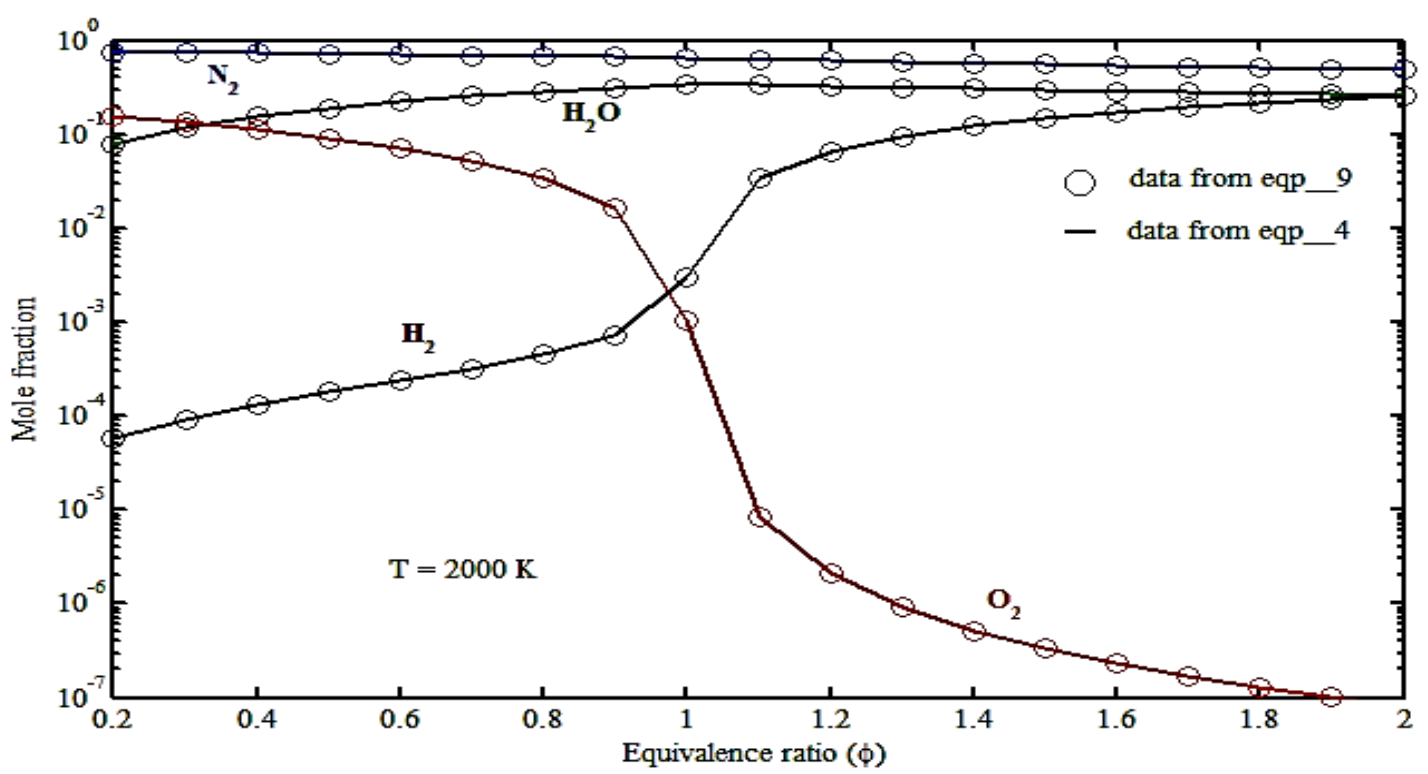

(a)

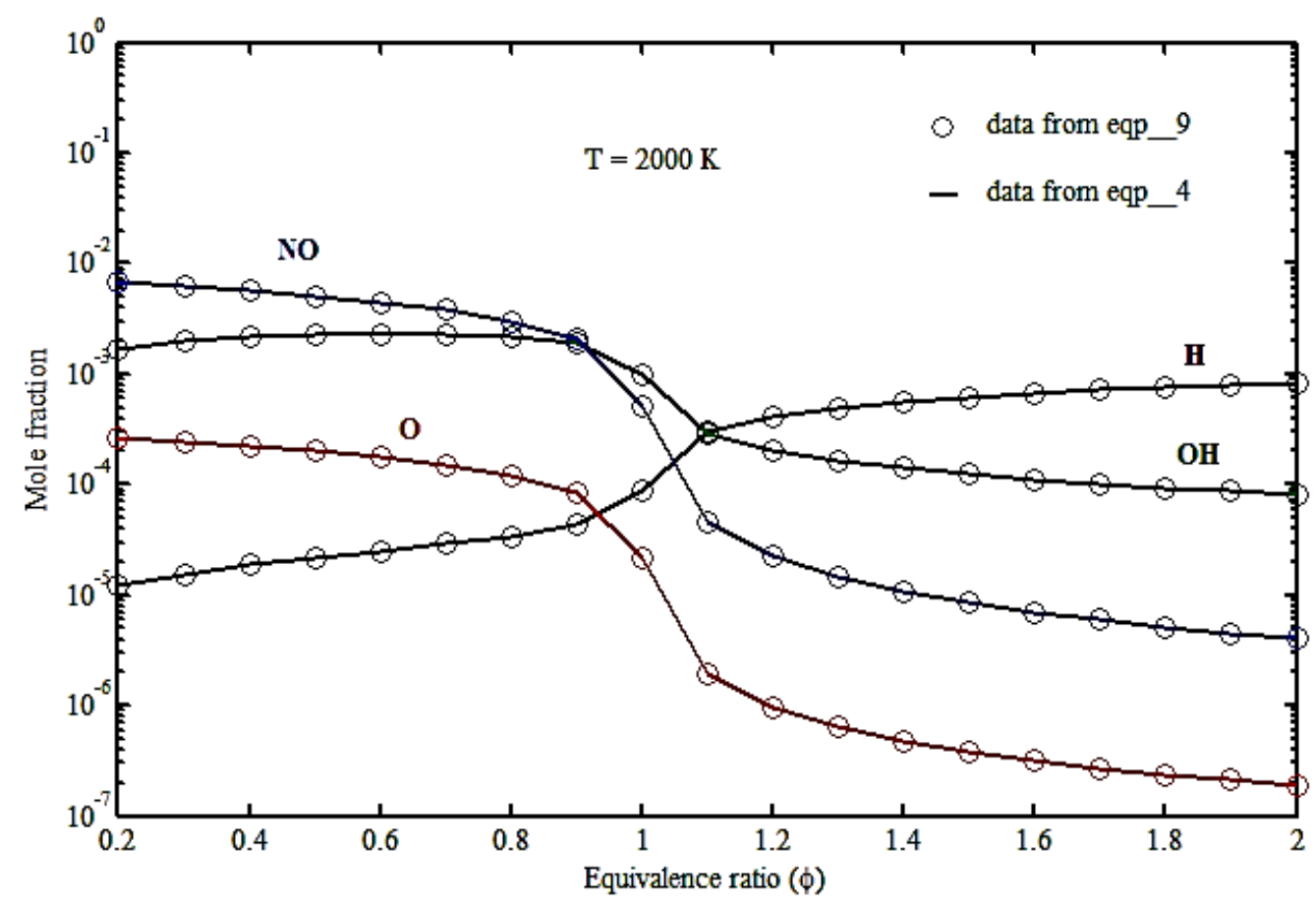

(b)

Figure 7. Comparison between eqp_9 and eqp_4: mole fraction as a function of $\phi$ at $2000 \mathrm{~K}$. 


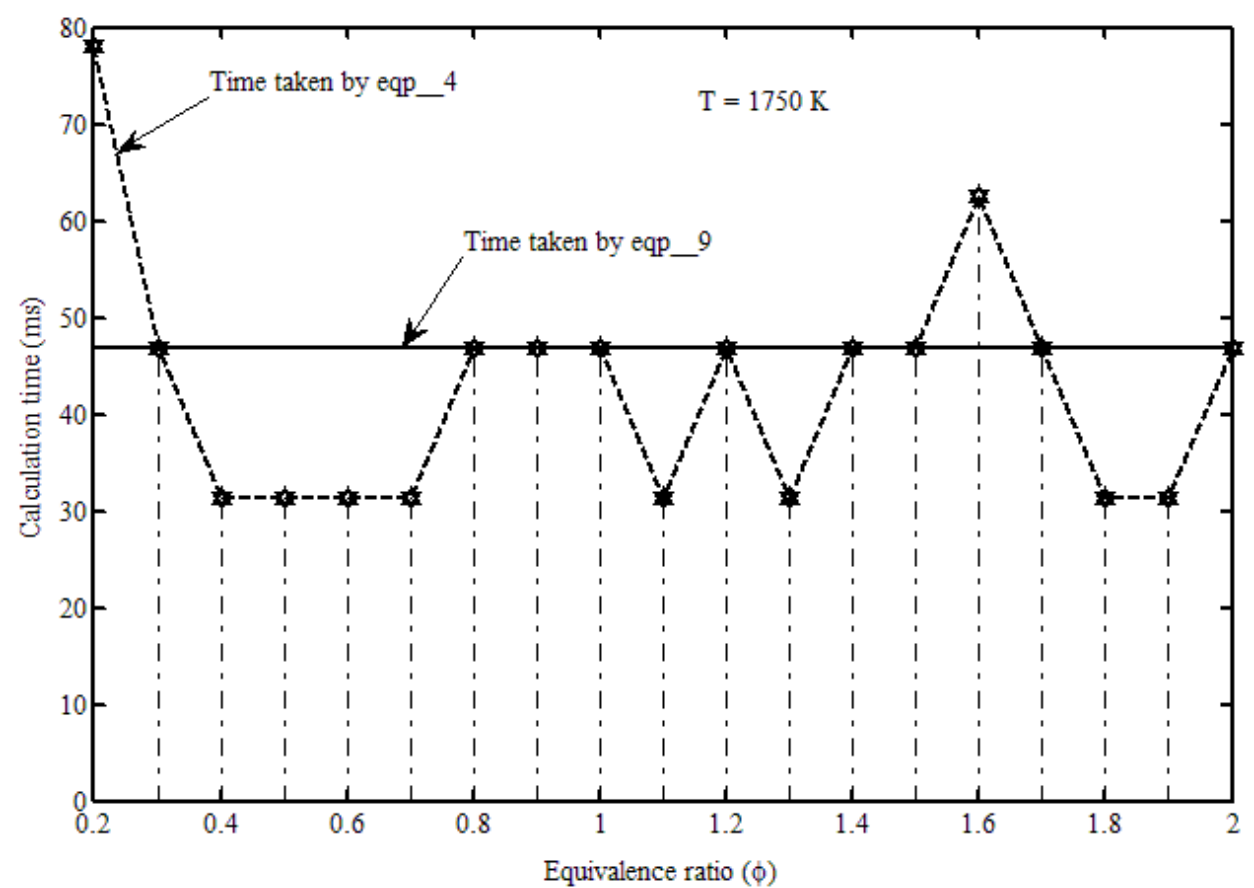

Figure 8. Comparison between the times consumed by eqp_9 and eqp_4 in mole fraction calculations.

\section{HydEnPro Mole Fraction Results}

Results illustrating composition shifts with $\phi$ are given in Figure 9 for hydrogen combustion at $p=30 \mathrm{~atm}$ and at two different temperatures, 1750 and $2250 \mathrm{~K}$. The mole fraction axis is drawn in logarithmic scale, as this scale gives a more meaningful picture. However, the results are for isooctane fuel $\left(\mathrm{C}_{8} \mathrm{H}_{18}\right)$ combustion. In our case, the components that contain carbon are absent from the product mixture. For the remaining constituents, the current results emphasize again the validity of HydEnPro. The mole fraction variations with $\phi$ are complicated phenomena.

The largest mole fractions are for $\mathrm{N}_{2}$ and $\mathrm{H}_{2} \mathrm{O}$. Comparison between the three parts of Figure 9 reveals that when the reaction temperature is increased, there is an exponential rise in product species such as $\mathrm{O}_{2}, \mathrm{H}_{2}, \mathrm{H}, \mathrm{O}, \mathrm{OH}$ and NO. In addition, the $\mathrm{O}_{2}$ fraction seems insensitive to temperature in the lean zone $(\phi<1)$, whereas the increase in $\phi$ leads to an increase in the $\mathrm{H}_{2}$ fraction and decreased $\mathrm{O}_{2}, \mathrm{O}, \mathrm{OH}$ and $\mathrm{NO}$ mole fractions. At the higher temperature of $2750 \mathrm{~K}$, there is a significant amount of nitric oxide (NO). If any gas in an engine cylinder is raised to this high temperature, that gas will tend toward equilibrium (Heywood, 1988). Since the chemistry for most species that contribute to the thermodynamic properties is fast enough for engine time scales, in many cases local equilibrium may be assumed. Nitric oxide, however, is significant even though its concentrations are relatively low because it is an air pollutant (Ferguson \& Kirkpatrick, 2001). 


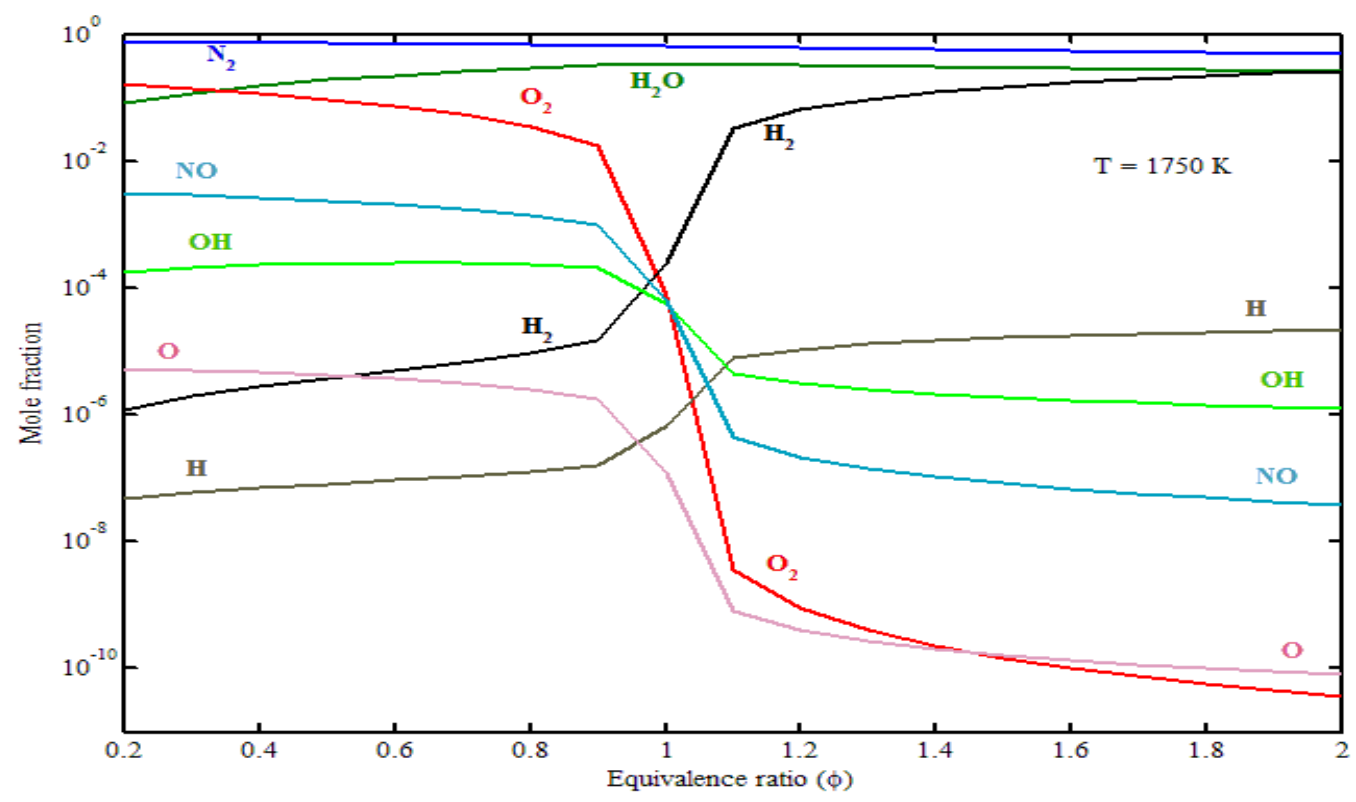

(a)

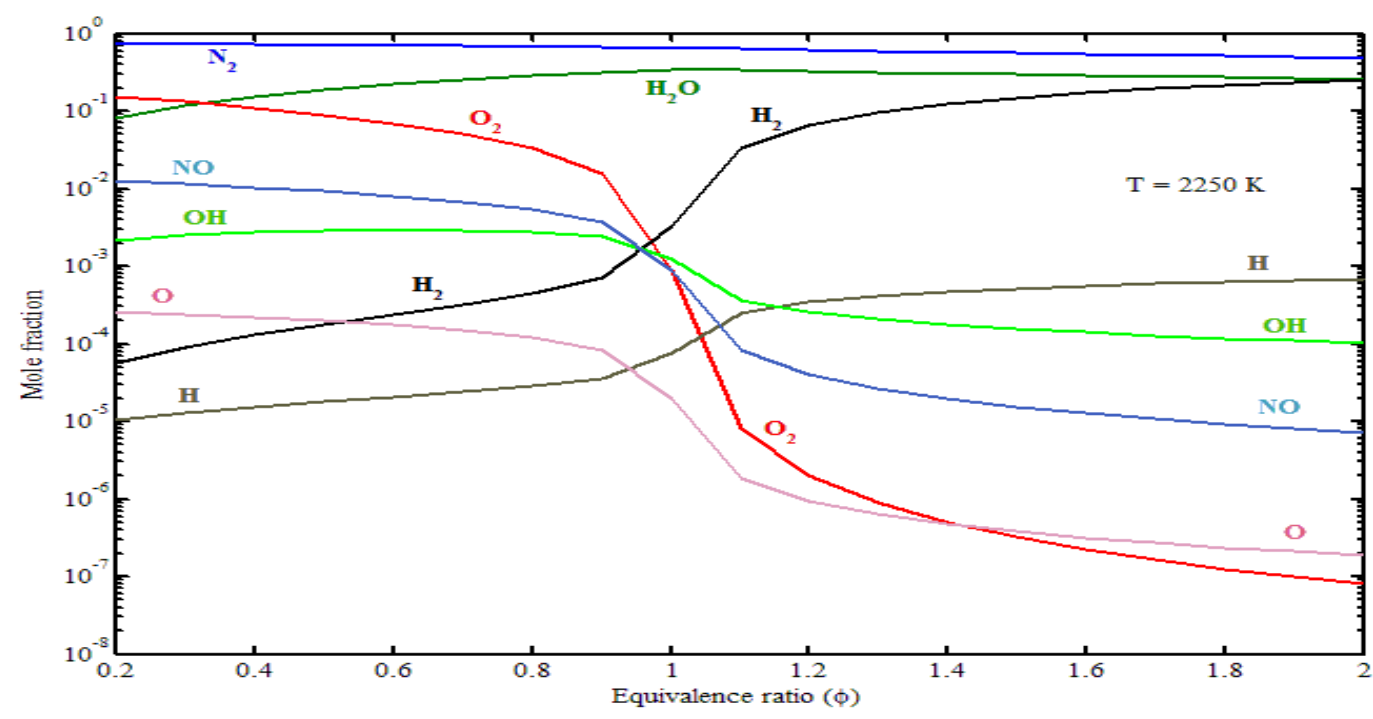

(b)

Figure 9. Mole fractions of equilibrium products as a function of fuel/air equivalence

\section{HydEnPro Properties Results}

Figure 10 presents the trends of the ratio $C_{p} / R, h$ and $s$ with temperature variation. The validity of HydEnPro is also checked herein by comparing the results of $C_{p} / R$ presented in this figure with those in the standard literature. The good concurrence emphasizes that HydEnPro translation for the data bases and its interfaces is correctly implemented. A closer look at Figure 10(a) reveals that its curves are ordered in three categories depending upon the number of atoms in each constituent: monatomic $(\mathrm{O}$ and $\mathrm{H})$, diatomic $\left(\mathrm{N}_{2}, \mathrm{O}_{2}, \mathrm{H}_{2}, \mathrm{OH}\right.$ and $\left.\mathrm{NO}\right)$ and triatomic $\left(\mathrm{H}_{2} \mathrm{O}\right)$. The triatomics have the greatest 
specific heat, followed by the diatomics, and, lastly the monatomics. Further, the triatomic components have a greater temperature dependence than the diatomic constituents. In comparison, the monatomic species have nearly constant specific heats over a wide range of temperatures. These facts were also indicated by Turns (2000). The enthalpy and entropy variations are swept with temperature for three different fuel/air equivalence ratios. A higher equivalence ratio leads to higher entropy product mixtures but with lower enthalpy. This is because richer mixtures increase some fractions and decrease others. The result is the trends shown. Figure 11 shows how the thermodynamic properties $\left(M, \gamma\right.$ and $\left.C_{p}\right)$ at chemical equlibrium vary with the equivalence ratio and temperature for hydrogen fuel combustion. Apart from the difference produced from the absence of carbon components, the standard figures are accurately reproduced, which shows that HydEnPro also correctly calculates the thermodynamic properties $M, C_{p}$, and $\gamma$.

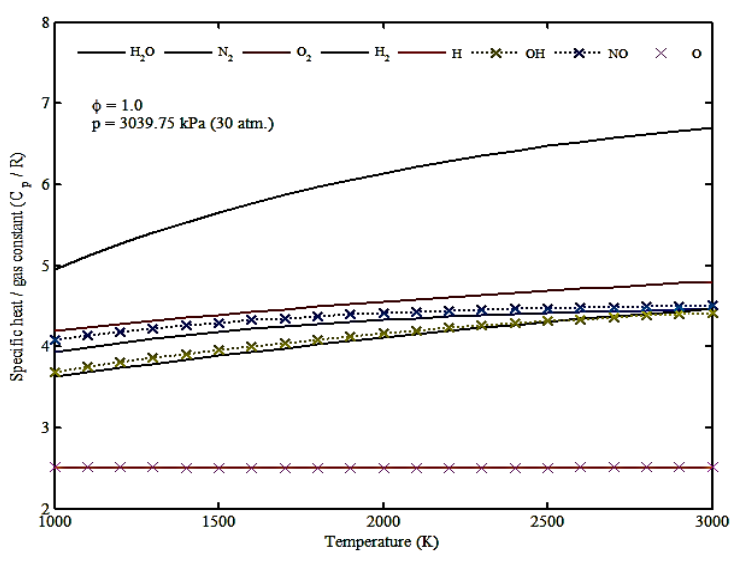

(a)

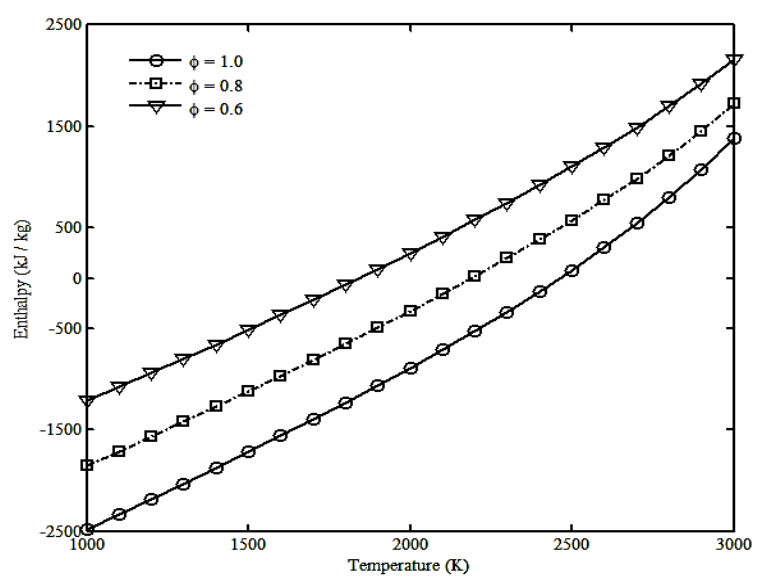

(b)

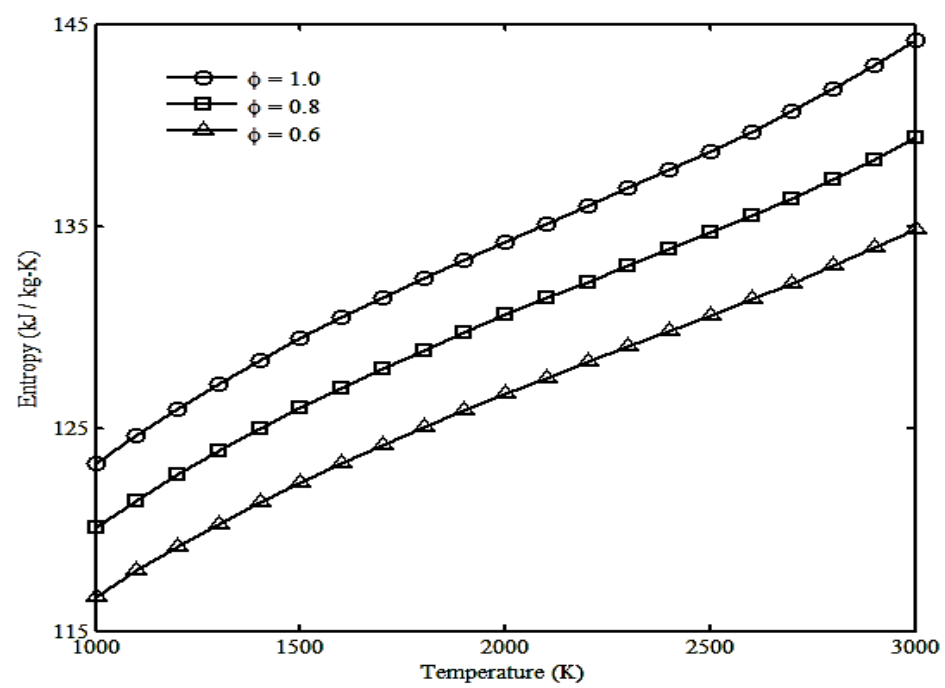

(c)

Figure 10. Variation of $C_{p}, h$, and $s$ of the burned gas with temperature at different fuel/air equivalence ratios. 


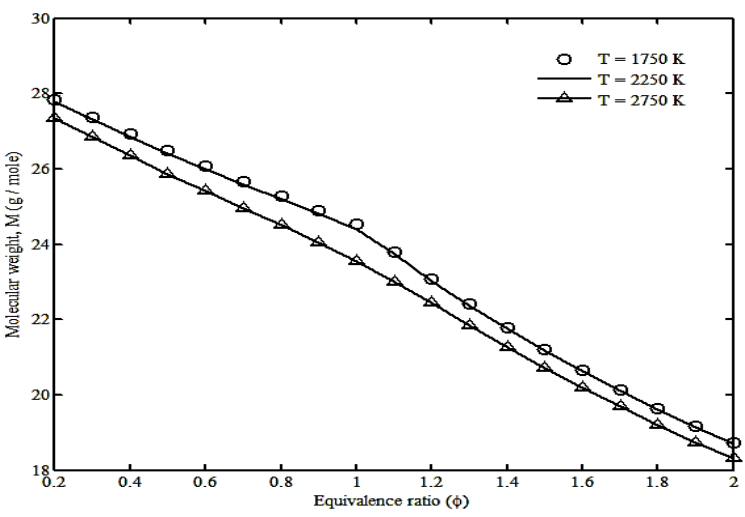

(a)

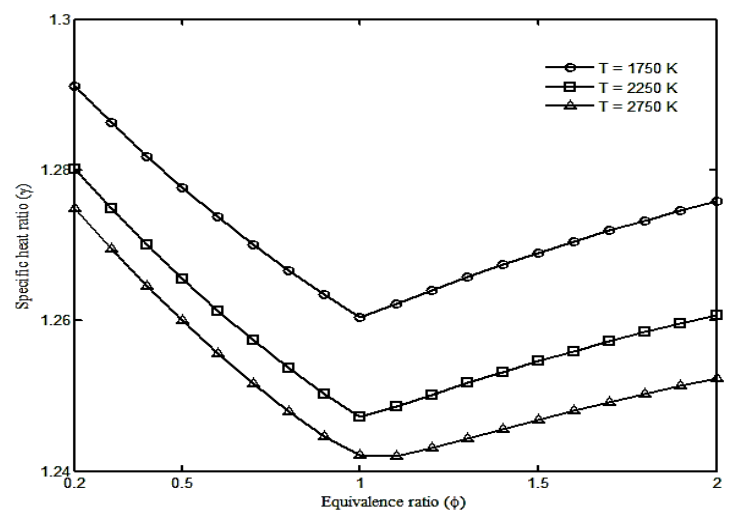

(b)

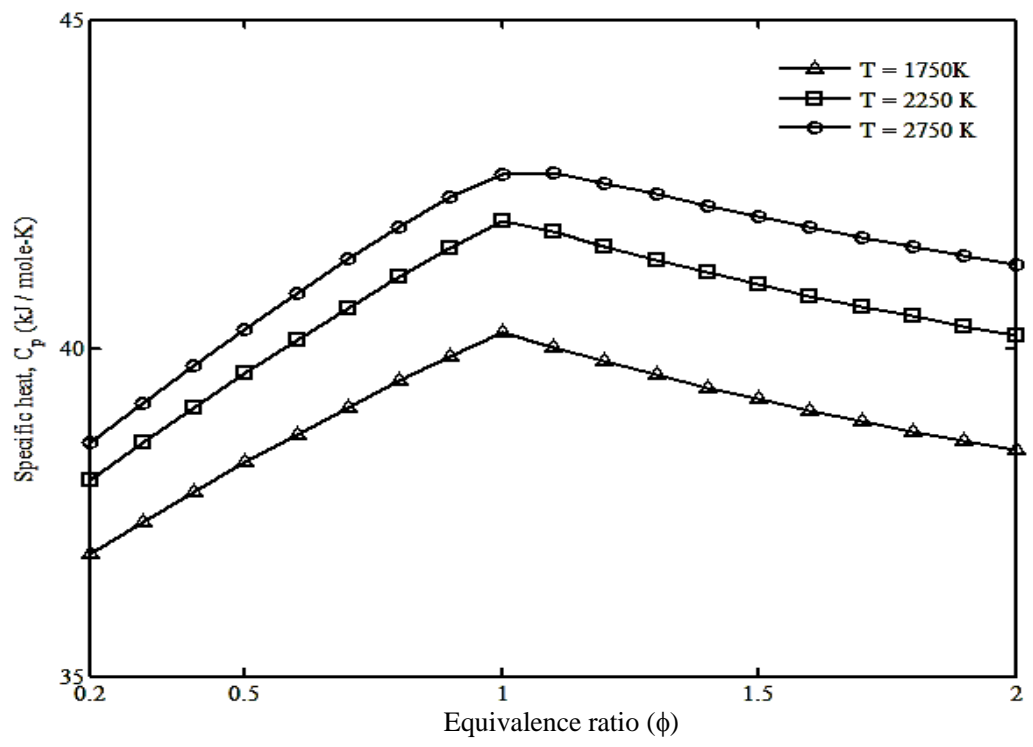

(c)

Figure 11. Variation of $M, \gamma$, and $C_{p}$ of the burned gas with $\phi$ at different temperatures.

\section{CONCLUSIONS}

The simulation model "HydEnPro" predicted the chemical equilibrium composition and thermodynamic properties for combustion reactants and combustion products. This model implemented in MATLAB is developed for predicting equilibrium compositions that occur in $\mathrm{H}_{2}$ ICEs. Evaluation with Chemkin tables showed excellent agreement. Using different thermodynamic tables yields insignificant differences. Additional validations showed that HydEnPro agrees well with what is shown in the standard literature.

\section{ACKNOWLEDGMENTS}

The authors would like to express their gratitude to Universiti Malaysia Pahang (UMP) for providing laboratory facilities and financial support (RDU110332). 


\section{REFERENCES}

AFTP (Adiabatic Flame Temperature Program) (2012). http://www.depcik.com/eduprograms/aftp.htm. (Accessed 18 October 2012).

Campbell, C. J. (2000). Myth of spare capacity setting the stage for another oil shock. Oil and Gas Journal, 20, 20-21.

Cruise, D. R. (1964). Notes on the rapid computation of chemical equilibria. Journal of Physical Chemistry, 68, 3797-3802.

Ferguson, C. R. \& Kirkpatrick, A. T. (2001). Internal combustion engine: applied thermodynamic (2nd ed.). New York: John Wiley.

Gaseq (2011). http://www.arcl02.dsl.pipex.com/. (Accessed 13 October 2011).

Gordon, S. \& McBride, B. J. (1976). Computer program for calculation of complex chemical equilibrium compositions, rocket performance, incident and reflected shocks, and Chapman-Jouguet detonations. NASA Technical Report No. SP273.

Hamada, K. I., Rahman, M. M., \& Aziz, A. R. A. (2012). Time-averaged heat transfer correlation for direct injection hydrogen fueled engine. International Journal of Hydrogen Energy, 37(24), 19146-19157.

Hamada, K. I., Rahman, M. M., Abdullah, M. A., Bakar, R. A., \& Aziz, A. R. A. (2013). Effect of mixture strength and injection timing on combustion characteristics of a direct injection hydrogen-fueled engine. International Journal of Hydrogen Energy, 38, 3793-3801.

Hamada, K. I., Rahman, M. M., and Aziz, A. R. A. (2012a). Characteristics of the timeaveraged overall heat transfer in a direct injection hydrogen fueled engine. International Journal of Hydrogen Energy, 38(11), 4816-4830.

Hamada, K. I., Rahman, M. M., and Aziz, A. R. A. (2012b). Time-averaged heat transfer correlation for direct injection hydrogen fueled engine. International Journal of Hydrogen Energy, 37(24), 19146-19157.

Heywood, J. B. (1988). Internal combustion engine fundamentals. New York: McGrawHill.

Kamil, M., Rahman, M. M., \& Bakar, R. A. (2011). Performance evaluation of external mixture formation strategy in hydrogen fueled engine. Journal of Mechanical Engineering and Sciences, 1, 87-98.

Kamil, M., Rahman, M. M., \& Bakar, R. A. (2012). Modeling of SI engine for dual fuels of hydrogen, gasoline and methane with port injection feeding system. Energy Education Science and Technology, 29(2), 1399-1416.

Kee, R. J., Rupley, F. M., \& Miller, J. A. (1991). The Chemkin thermodynamic data base. Sandia Technical Report No. SAND87-8215B.

Kelly, N. A., Gibson, L., Cai, M., Spearot, J., \& Ouwerkerk, D. B. (2010). Development of a renewable hydrogen economy: optimization of existing technologies. International Journal of Hydrogen Energy, 35, 892-899.

Lee, J. Y., Yoo, M., Cha, K., Lim, T. W., \& Hur, T. (2009). Life cycle cost analysis to examine the economical feasibility of hydrogen as an alternative fuel. International Journal of Hydrogen Energy, 34, 4243-4255.

NBS (National Bureau of Standards) (1971). JANAF Thermochemical Tables. NBS Technical Report No. NSRDS-NBS37.

Olikara, C. \& Borman, G. L. (1975). A computer program for calculating properties of equilibrium combustion products with some applications to IC engines. SAE Technical Paper No. 750468. 
Pfeiffer, D. A. (2006). Eating fossil fuels: oil, food, and the coming crisis in agriculture. Canada: New Society Publisher.

Rahman, M. M., Mohammed, M. K., \& Bakar, R. A. (2009a). Trends of rotational speed on engine performance for four-cylinder direct injection hydrogen fueled engine. Trends of Applied Sciences and Research, 4(4), 188-199.

Rahman, M. M., Mohammed, M. K., \& Bakar, R. A. (2009b). Air fuel ratio on engine performance and instantaneous behaviour of crank angle for four cylinder direct injection hydrogen fueled engine. Journal of Applied Sciences, 9(16), 28772886.

Rahman, M. M., Hamada, K. I., Noor, M. M., Kadirgama, K., Maleque, M. A., \& Bakar, R. A. (2010). Heat transfer characteristics of intake port for spark ignition engine: A comparative study. Journal of Applied Sciences, 10(18), 2019-2026.

Rahman, M. M., Kamil, M., \& Bakar, R. A. (2011a). Engine performance and optimum injection timing for 4-cylinder direct injection hydrogen fueled engine. Simulation Modelling Practice and Theory, 19, 1734-1751.

Rahman, M. M., Hamada, K. I., \& Kadirgama, K. (2011b). Heat transfer of intake port for hydrogen fueled port injection engine: A steady state approach. International Journal of Physical Sciences, 6(16), 4036-4043.

Rahman, M. M., Hamada, K. I. and Aziz, A. R. A. (2013). Effect of mixture strength and injection timing on combustion characteristics of a direct injection hydrogen-fueled engine. International Journal of Hydrogen Energy, (in press). http://dx.doi.org/10.1016/j.ijhydene.2013.01.136.

Smith, W. R. \& Missen, R. W. (1968). Calculating complex chemical equilibria by an improved reaction-adjustment method. Canadian Journal of Chemical Engineering, 46, 263-272.

Sopena, C., Dieguez, P. M., Sainz, D., Urroz, J. C., Guelbenzu, E., \& Gandia, L. M. (2010). Conversion of a commercial spark ignition engine to run on hydrogen: Performance comparison using hydrogen and gasoline. International Journal of Hydrogen Energy, 35, 1420-1429.

Turns, S. R. (2000). An introduction to combustion: concepts and applications (2nd ed.). Boston: McGraw-Hill.

Turns, S. R. (2006). Thermal sciences: an integrated approach. New York: Cambridge University Press.

Verhelst, S. \& Wallner, T. (2009). Hydrogen-fueled internal combustion engines. Progress in Energy and Combustion Science, 35, 490-527.

Villars, D. S. (1959). A method of successive approximations for computing combustion equilibria on high speed digital computers. Journal of Physical Chemistry, 63, 521-525. 